\title{
Governing equations and numerical solutions of tension leg platform with finite amplitude motion *
}

\author{
ZENG Xiao-hui (曾晓辉)， SHEN Xiao-peng (沈晓鹏)，WU Ying-xiang (吴应湘) \\ (Division of Engineering Sciences, Institute of Mechanics, Chinese Academy of Sciences, \\ Beijing 100080, P. R. China)
}

(Communicated by LI Jia-chun)

\begin{abstract}
It is demonstrated that when tension leg platform (TLP) moves with finite amplitude in waves, the inertia force, the drag force and the buoyancy acting on the platform are nonlinear functions of the response of TLP. The tensions of the tethers are also nonlinear functions of the displacement of TLP. Then the displacement, the velocity and the acceleration of TLP should be taken into account when loads are calculated. In addition, equations of motions should be set up on the instantaneous position. A theoretical model for analyzing the nonlinear behavior of a TLP with finite displacement is developed, in which multifold nonlinearities are taken into account, i.e., finite displacement, coupling of the six degrees of freedom, instantaneous position, instantaneous wet surface, free surface effects and viscous drag force. Based on the theoretical model, the comprehensive nonlinear differential equations are deduced. Then the nonlinear dynamic analysis of ISSC TLP in regular waves is performed in the time domain. The degenerative linear solution of the proposed nonlinear model is verified with existing published one. Furthermore, numerical results are presented, which illustrate that nonlinearities exert a significant influence on the dynamic responses of the TLP.
\end{abstract}

Key words tension leg platform (TLP), finite displacement, nonlinear dynamic response, numerical solution, wave loads

Chinese Library Classification P732, U674

2000 Mathematics Subject Classification 76B15

Digital Object Identifier(DOI) 10.1007/s 10483-007-0105-1

\section{Introduction}

As the development of the national economy with high speed, the demand for oil and natural gas in China has been continuously augmenting. And the gaps between supply and demand become increasingly wider. In China, the import volume of crude oil reached 120 million tons in 2004, intending to increase $17 \%$ in 2005 . The oil import channel is prone to be interdicted for diversified variable political, economical and security factors. Therefore, the domestic rational exploitation should be strengthened. However, the oil and gas resource in land and inshore continental shelf gradually decrease. In view of this situation, the petroleum industries in China take great interest in deep water exploration and development. The exploitation platforms fit for shallow water are not suitable to deep sea because the construction cost increase greatly with the water depth. To meet the challenge, the concept of deep-sea platform is introduced.

Tension leg platform (TLP) is a typical compliant floating working station for oil exploitation in deep sea, as shown in Fig.1. It consists of hull, taut tethers and foundations, which

\footnotetext{
* Received Mar.13, 2006; Revised Oct.10, 2006

Project supported by "Creativeness Project of the Tenth Five-Year Plan" of Chinese Academy of Sciences (No.KJCX2-SW-L03) and the National High-Tech Research and Development Program of China (863 Program) (No.2004AA617010)

Corresponding author ZENG Xiao-hui, Associate Professor, Doctor, E-mail: zxh@imech.ac.cn
} 
allows motions of surge, sway, and yaw in the horizontal plane and heave, pitch, and roll in the vertical plane. The dynamic response of TLP is an important problem of offshore mechanics, and there are many researches on it. Williams and Rangappa ${ }^{[1]}$ developed an approximate semi-analytical technique to calculate hydrodynamic loads and added mass and damping coefficients for idealized TLP consisting of arrays of circular cylinder. Yilmaz ${ }^{[2]}$ presented an exact analytical method to solve the diffraction and radiation problems of a group of cylinders, taking account of the interaction between the cylinders. Yilmaz, Incecik and Barltrop ${ }^{[3]}$ calculated free surface elevations for an array of four cylinders. Ahmad ${ }^{[4]}$ conducted response analysis considering viscous hydrodynamic force, variable added mass and large excursion. In addition, Ahmad, Islam and Ali ${ }^{[5]}$ investigated TLP' s sensitivity to dynamic effects of the wind. Chandrasekaran and Jain ${ }^{[6,7]}$ proposed a triangular configuration TLP, and developed a method to analyze the dynamic behavior of triangular and square TLP. Furthermore, they performed numerical studies to compare the dynamic responses of a triangular TLP with that of a square TLP.

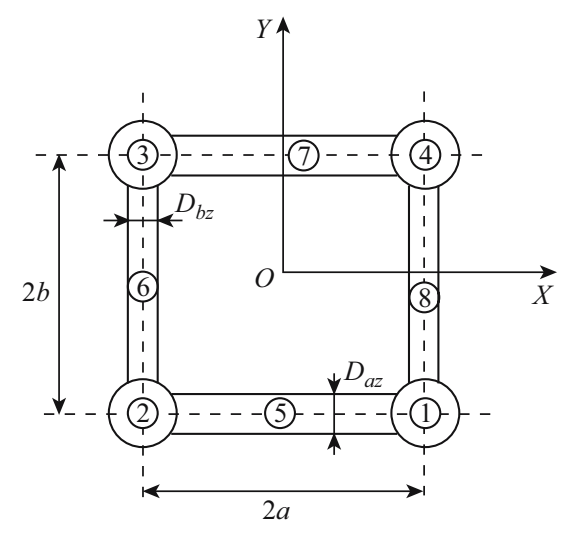

(a) Planform of TLP

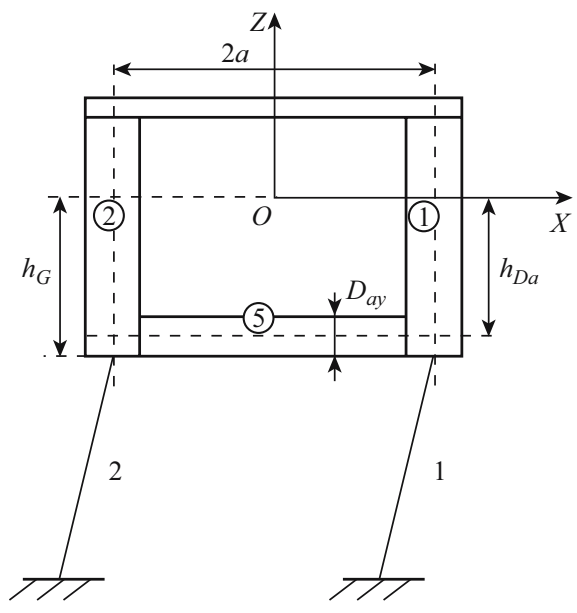

(c) Front view of TLP



(b) The coordinate systems



(d) Side view of TLP

Fig.1 Sketch of TLP and the coordinate systems

Up to the author's knowledge, the existing investigations on TLP mostly make a priori assumptions explicitly or implicitly that the translational displacements and angular displace- 
ments being kept infinitesimal. Therefore, the finite motions and accordingly aroused other nonlinear factors are not taken into account. In fact, in severe sea state, swell dominant sea state or adverse operation state, the displacements of TLP may be large quantity and should not be taken for infinitesimal. Very few investigations ostensibly claim to have considered arbitrary displacements. However, it may not be the fact. The reason is that, when they deduce the stiffness matrix, arbitrary displacement is given just in one direction while keeping all other degrees of freedom restrained. In nature, stiffness matrix obtained by such technique is with respect to the initial static equilibrium position. This technique can only be employed for linear problem. For nonlinear problem, the stiffness matrix should be deduced with reference to the instantaneous displaced position (i.e., the structure may move in all six degrees of freedom, none of them should be restrained).

The aforementioned assumptions make the process of dynamic analysis of TLP fairly easy. However, such technique places too severe restriction to include all load cases, especially in some extreme circumstances. It is obvious that the method can be modified to adapt for more general operation state if we abandon the infinitesimal assumptions. Finite displacements are visible nonlinearities. In addition, there are more concomitant nonlinear factors induced by finite displacements. For example, the six degrees of freedom are coupled; the hydrodynamic forces on TLP are response dependent (i.e., wave forces are functions of the instantaneous position, velocity, acceleration and wet surface of TLP). Although considering all the nonlinearities makes the problem very complex, it is worthwhile according to our analysis. We have investigated the dynamic response of a floating circular cylinder with a taut tether taking account of all the nonlinearities above-mentioned ${ }^{[8]}$. It is shown that these nonlinear factors exert a significant influence on the dynamic responses of the tethered cylinder.

Whereas the reason we interpret above, this paper investigates the nonlinear dynamic response of a typical TLP. The nonlinearities include finite displacements, coupling of the six degrees of freedom, instantaneous position, instantaneous wet surface, free surface effects and viscous drag force.

In this paper, the major assumptions are made as follows: (i) The motion of cylinder is finite instead of infinitesimal. (ii) The cylinder is assumed sufficient slender, and then the wave diffraction effects have been neglected (For severe or swell sea state, the low frequency components are dominant, and then the wave lengths are large enough to satisfy this assumption). (iii) Wave forces are evaluated at the instantaneous displaced position of the cylinder by Morison's equation. (iv) The free surface effects are taken into account.

\section{Derivation of external force and equations of motion}

A typical TLP consisting of four columns and pontoons is shown in Figs.1(a), (c) and (d). Three right-hand Cartesian coordinate systems oxyz, $O X Y Z, G \xi \eta \zeta$ are defined in Fig.1(b). The oxyz is space fixed coordinate system, plane oxy coincides with the undisturbed calm water surface, and the positive $z$-axis is pointing upwards. This coordinate system is used to define wave. The $O X Y Z$ is also space fixed coordinate system, which has its origin located at the gravity center (C.G.) of the undisturbed TLP. Three axes of coordinate system $O X Y Z$ are in parallel with those of oxyz. The $G \xi \eta \zeta$ is body fixed coordinate system, which coincides with the $O X Y Z$ when the TLP has zero displacement. The motions of TLP are denoted by the displacements $X_{1}, X_{2}, X_{3}, X_{4}, X_{5}$ and $X_{6}$ of $G \xi \eta \zeta$ with respect to $O X Y Z . X_{1}, X_{2}, X_{3}$ are the coordinates of $G$ in $O X Y Z$, which denote the translation of TLP. The longitudinal displacement $X_{1}$ is defined as surge, the transverse displacement $X_{2}$ is sway, and the vertical one $X_{3}$ along $Z$ is heave. Angular motions are represented in terms of three Eulerian angles $X_{4}, X_{5}, X_{6}$ of $G \xi \eta \zeta$ with reference to $O X Y Z$. In this paper, $X_{1}, X_{2}, X_{3}, X_{4}, X_{5}$ and $X_{6}$ are finite magnitude instead of infinitesimal. 
The transformation of coordinates can be written as follows:

$$
\left(\begin{array}{l}
X \\
Y \\
Z
\end{array}\right)=\left(\begin{array}{l}
X_{1} \\
X_{2} \\
X_{3}
\end{array}\right)+\left(\begin{array}{lll}
t_{11} & t_{12} & t_{13} \\
t_{21} & t_{22} & t_{23} \\
t_{31} & t_{32} & t_{33}
\end{array}\right)\left(\begin{array}{l}
\xi \\
\eta \\
\zeta
\end{array}\right),
$$

where,

$$
\begin{aligned}
& \left(\begin{array}{lll}
t_{11} & t_{12} & t_{13} \\
t_{21} & t_{22} & t_{23} \\
t_{31} & t_{32} & t_{33}
\end{array}\right) \\
= & \left(\begin{array}{ccc}
\cos X_{5} \cos X_{6} & -\cos X_{5} \sin X_{6} & \sin X_{5} \\
\sin X_{4} \sin X_{5} \cos X_{6}+\cos X_{4} \sin X_{6} & -\sin X_{4} \sin X_{5} \sin X_{6}+\cos X_{4} \cos X_{6} & -\sin X_{4} \cos X_{5} \\
-\cos X_{4} \sin X_{5} \cos X_{6}+\sin X_{4} \sin X_{6} & \cos X_{4} \sin X_{5} \sin X_{6}+\sin X_{4} \cos X_{6} & \cos X_{4} \cos X_{5}
\end{array}\right) .
\end{aligned}
$$

If the angles are assumed to be infinitesimal, and the components of the matrix are truncated after the first order small magnitude, Eq.(1b) can be linearized as

$$
\left(\begin{array}{lll}
t_{11} & t_{12} & t_{13} \\
t_{21} & t_{22} & t_{23} \\
t_{31} & t_{32} & t_{33}
\end{array}\right)=\left(\begin{array}{ccc}
1 & -X_{6} & X_{5} \\
X_{6} & 1 & -X_{4} \\
-X_{5} & X_{4} & 1
\end{array}\right) .
$$

Generally, the current method employs Eq.(1c) to analyze the overall dynamic response of TLP. It can be found that Eq.(1b) differs from Eq.(1c) in many ways. The latter is the approximation of the former. In addition, the latter ignores some terms related to couplings among various degrees of freedom. In view of Eq.(1a) and Eq.(1b), we can see that coordinates $Y$ and $Z$ of TLP in $O X Y Z$ are functions of $X_{4}, X_{5}, X_{6}$, whereas $Y$ and $Z$ are merely functions of $X_{4}, X_{6}$ and $X_{4}, X_{5}$, respectively, if Eq.(1a) and Eq.(1c) are adopted. As the angular displacements are finite magnitude, such approximation will obviously induce comparatively large errors. Even if the rotation is small, those coupled terms may evoke considerable effect in view of the small damping and the near-resonance state.

In this paper, we study the situation of finite displacements with the exact transformation matrix (1b). Thus the interactions between degrees of freedom can be retained, and the precision of numerical calculation can be improved.

By using Newton's second law, we can obtain the equations of six components $X_{i}$ of TLP motions as

$$
\left(\begin{array}{cccccc}
M & 0 & 0 & 0 & 0 & 0 \\
0 & M & 0 & 0 & 0 & 0 \\
0 & 0 & M & 0 & 0 & 0 \\
0 & 0 & 0 & I_{1} & 0 & 0 \\
0 & 0 & 0 & 0 & I_{2} & 0 \\
0 & 0 & 0 & 0 & 0 & I_{3}
\end{array}\right)\left(\begin{array}{c}
\ddot{X}_{1} \\
\ddot{X}_{2} \\
\ddot{X}_{3} \\
\dot{\omega}_{1} \\
\dot{\omega}_{2} \\
\dot{\omega}_{3}
\end{array}\right)=\left(\begin{array}{c}
F_{1}\left(X_{i}, \dot{X}_{i}, \ddot{X}_{i}\right) \\
F_{2}\left(X_{i}, \dot{X}_{i}, \ddot{X}_{i}\right) \\
F_{3}\left(X_{i}, \dot{X}_{i}, \ddot{X}_{i}\right) \\
F_{4}\left(X_{i}, \dot{X}_{i}, \ddot{X}_{i}\right)-\left(I_{3}-I_{2}\right) \omega_{2} \omega_{3} \\
F_{5}\left(X_{i}, \dot{X}_{i}, \ddot{X}_{i}\right)-\left(I_{1}-I_{3}\right) \omega_{3} \omega_{1} \\
F_{6}\left(X_{i}, \dot{X}_{i}, \ddot{X}_{i}\right)-\left(I_{2}-I_{1}\right) \omega_{1} \omega_{2}
\end{array}\right),
$$

in which $M$ is the body mass of TLP in air, and $I_{i}(i=1,2,3)$ are the moments of inertia with respect to the principal axes through C.G. $F_{i}$ are the components of external force $(i=1,2,3)$ and moment $(i=4,5,6)$ vectors, respectively. $\omega_{i}(i=1,2,3)$ are the components of angular velocity, and the dot over variable means time derivative. Angular velocities are given by

$$
\begin{aligned}
& \omega_{1}=\dot{X}_{4} \cos X_{5} \cos X_{6}+\dot{X}_{5} \sin X_{6}, \\
& \omega_{2}=-\dot{X}_{4} \cos X_{5} \sin X_{6}+\dot{X}_{5} \cos X_{6}, \\
& \omega_{3}=\dot{X}_{4} \sin X_{5}+\dot{X}_{6},
\end{aligned}
$$




$$
\begin{aligned}
\dot{\omega}_{1}= & \ddot{X}_{4} \cos X_{5} \cos X_{6}-\dot{X}_{4} \dot{X}_{5} \sin X_{5} \cos X_{6}-\dot{X}_{4} \dot{X}_{6} \cos X_{5} \sin X_{6} \\
& +\ddot{X}_{5} \sin X_{6}+\dot{X}_{5} \dot{X}_{6} \cos X_{6}, \\
\dot{\omega}_{2}= & -\ddot{X}_{4} \cos X_{5} \sin X_{6}+\dot{X}_{4} \dot{X}_{5} \sin X_{5} \sin X_{6}-\dot{X}_{4} \dot{X}_{6} \cos X_{5} \cos X_{6} \\
& +\ddot{X}_{5} \cos X_{6}-\dot{X}_{5} \dot{X}_{6} \cos X_{6}, \\
\dot{\omega}_{3}= & \ddot{X}_{4} \sin X_{5}+\dot{X}_{4} \dot{X}_{5} \cos X_{5}+\ddot{X}_{6} .
\end{aligned}
$$

If angular displacements are small magnitude of the first order, Eqs.(3)-(5) and Eqs.(3a)-(5a) can be simplified as

$$
\begin{aligned}
& \left(\begin{array}{lll}
\omega_{1} & \omega_{2} & \omega_{3}
\end{array}\right)=\left(\begin{array}{lll}
\dot{X}_{4} & \dot{X}_{5} & \dot{X}_{6}
\end{array}\right) \\
& \left(\begin{array}{llll}
\dot{\omega}_{1} & \dot{\omega}_{2} & \dot{\omega}_{3}
\end{array}\right)=\left(\begin{array}{lll}
\ddot{X}_{4} & \ddot{X}_{5} & \ddot{X}_{6}
\end{array}\right) .
\end{aligned}
$$

Comparing these two equations with Eqs.(3)-(5a), we can easily see that many coupling terms emerge if finite displacements are considered.

The external forces and moments $F_{i}(i=1,2, \cdots, 6)$ in the right hand of Eq.(2) are coupled with the instantaneous response of TLP, which is different from the case with small motion. In that case, hydrodynamic forces are independent of response while the hydrostatic forces and the tension of tendon are linear functions of displacements.

Then we will derive the external forces and moments. From the process of derivation, we can see that external forces and moments are assuredly coupled with the instantaneous position, velocity and acceleration of TLP. They are nonlinear functions of the response.

\subsection{External forces and moments vectors acting on TLP}

TLP endures tension of tendons, hydrodynamic and hydrostatic forces acting on columns and pontoons and the self gravities. After doing vector sums of those forces, we can obtain the principal vector $\boldsymbol{F}$ of external forces acting on TLP as follows:

$$
\boldsymbol{F}=\boldsymbol{F}_{\mathrm{w}}+\boldsymbol{F}_{\mathrm{B}}+\boldsymbol{F}_{\mathrm{Ot}}-M g \boldsymbol{k}=F_{1} \boldsymbol{i}+F_{2} \boldsymbol{j}+F_{3} \boldsymbol{k},
$$

where $\boldsymbol{i}, \boldsymbol{j}, \boldsymbol{k}$ are base vectors of system $O X Y Z, g$ is the acceleration of gravity. Similarly, we can get the resultant moment $M$ by summing external moment vectors together,

$$
\boldsymbol{M}=\boldsymbol{M}_{G \mathrm{w}}+\boldsymbol{M}_{G \mathrm{~B}}+\boldsymbol{M}_{G \mathrm{t}}=F_{4} \boldsymbol{e}_{1}+F_{5} \boldsymbol{e}_{2}+F_{6} \boldsymbol{e}_{3},
$$

where $\boldsymbol{e}_{1}, \boldsymbol{e}_{2}, \boldsymbol{e}_{3}$ are base vectors of system $G \xi \eta \zeta$. As said before, $F_{i}(i=1,2, \cdots, 6)$ are nonlinear functions of the response of TLP. As long as the formulas for calculating $F_{i}$ are obtained, we can perform evaluation of Eq.(2). The detailed formulas are given below.

\subsection{Hydrodynamic forces vectors}

When the circular cylinder moves to an arbitrary position in waves, the axis of cylinder may be inclined instead of vertically upwards. Then forces acting on the cylinder need to be written in terms of the normal components (with respect to the axis of the cylinder) of fluid acceleration, relative acceleration and velocity vectors between water particle and structural element. The relative acceleration and velocity vectors are functions of the response of TLP, and the fluid accelerations are calculated at the displaced position of TLP. Then one can easily see that the forces and responses are coupled.

In this paper, we present the formulae for calculation of hydrodynamic forces acting on column 1. The formulae for hydrodynamic forces on other columns and pontoons are similar. Using modified Morison equation ${ }^{[9]}$, force vector per unit length of arbitrary oriented cylinder $\boldsymbol{f}_{n}$ is written as

$$
\boldsymbol{f}_{n}=\rho \frac{\pi D^{2}}{4} \dot{\boldsymbol{V}}_{\mathrm{n}}+C_{a} \rho \frac{\pi D^{2}}{4} \dot{\boldsymbol{V}}_{\mathrm{rn}}+C_{d} \frac{\rho D}{2}\left|\boldsymbol{V}_{\mathrm{rn}}\right| \boldsymbol{V}_{\mathrm{rn}},
$$


in which $\rho$ is mass density of water, $D$ is the diameter of the cylinder, $C_{\mathrm{a}}$ is added mass coefficient, $C_{\mathrm{d}}$ is drag coefficient, $\dot{\boldsymbol{V}}_{\mathrm{n}}$ is acceleration vector of water particle normal to inclined cylinder, $\dot{\boldsymbol{V}}_{\text {rn }}$ and $\boldsymbol{V}_{\text {rn }}$ are relative acceleration and velocity vectors between the water particle and structural element normal to inclined column. The normal acceleration vector $\dot{\boldsymbol{V}}_{\mathrm{n}}$ is given as

$$
\dot{\boldsymbol{V}}_{\mathrm{n}}=\boldsymbol{e}_{3} \times\left(\dot{\boldsymbol{V}} \times \boldsymbol{e}_{3}\right) .
$$

$\dot{\boldsymbol{V}}$ is the fluid acceleration vector of water particle at the instantaneous position of column 1 , which is evaluated in reference frame oxyz employing modified Airy's linear wave theory with "stretching method" Chakrabarti ${ }^{[10]}$ used. The position of TLP always changes, and then the accelerations of fluid particle used to calculate wave forces also alter along with TLP. Hence one can see that

$$
\dot{\boldsymbol{V}}=\dot{\boldsymbol{V}}\left(X_{i}\right) \quad(i=1,2, \cdots, 6) .
$$

$\boldsymbol{e}_{3}$ is the unit vector along the Cartesian coordinate axis $G \zeta$, and it is the function of displacement of TLP,

$$
\boldsymbol{e}_{3}=\boldsymbol{e}_{3}\left(X_{i}\right) \quad(i=1,2, \cdots, 6) .
$$

Therefore, it is obvious that:

$$
\dot{\boldsymbol{V}}_{\mathrm{n}}=\dot{\boldsymbol{V}}_{\mathrm{n}}\left(X_{i}\right) \quad(i=1,2, \cdots, 6) .
$$

Combining Eqs.(9)-(11), we can see that $\dot{\boldsymbol{V}}_{\mathrm{n}}$ is nonlinear function of the displacement $X_{i}$. Similarly, relative velocity and acceleration vectors $\left(\boldsymbol{V}_{\text {rn }}\right.$ and $\left.\dot{\boldsymbol{V}}_{\text {rn }}\right)$ between the water particle and structural element normal to inclined column 1 can be given as

$$
\begin{aligned}
& \boldsymbol{V}_{\mathrm{rn}}=\boldsymbol{e}_{3} \times\left(\boldsymbol{V}_{\mathrm{r}} \times \boldsymbol{e}_{3}\right), \\
& \boldsymbol{V}_{\mathrm{rn}}=\boldsymbol{V}_{\mathrm{rn}}\left(X_{i}, \dot{X}_{i}\right) \quad(i=1,2, \cdots, 6), \\
& \dot{\boldsymbol{V}}_{\mathrm{rn}}=\boldsymbol{e}_{3} \times\left(\dot{\boldsymbol{V}}_{\mathrm{r}} \times \boldsymbol{e}_{3}\right)=\dot{\boldsymbol{V}}_{\mathrm{rn}}\left(X_{i}, \dot{X}_{j}, \ddot{X}_{i}\right) \quad(i=1,2, \cdots, 6 ; \quad j=4,5,6),
\end{aligned}
$$

in which, $\boldsymbol{V}_{\mathrm{r}}=\boldsymbol{V}-\boldsymbol{V}_{\mathrm{s}}, \dot{\boldsymbol{V}}_{\mathrm{r}}=\dot{\boldsymbol{V}}-\dot{\boldsymbol{V}}_{\mathrm{s}}, \boldsymbol{V}$ is the velocity vector of water particle, $\boldsymbol{V}_{\mathrm{s}}$ is the velocity vector of some point on the axis of column 1 . By analogy with that of $\dot{\boldsymbol{V}}_{\mathrm{n}}$, we can see that $\boldsymbol{V}_{\text {rn }}$ is nonlinear function of the displacement $X_{i}$ and velocity $\dot{X}_{i}$ of TLP, and $\dot{\boldsymbol{V}}_{\text {rn }}$ is nonlinear function of the displacement $X_{i}$, the velocity $\dot{X}_{i}$ and the acceleration $\ddot{X}_{i}$ of TLP.

As $\dot{\boldsymbol{V}}_{\mathrm{n}}, \dot{\boldsymbol{V}}_{\mathrm{rn}}, \boldsymbol{V}_{\mathrm{rn}}$ are all nonlinear functions of the dynamic responses of TLP, the wave force vector (Eq.(8)) $\boldsymbol{f}_{\mathrm{n}}=\boldsymbol{f}_{\mathrm{n}}\left(X_{i}, \dot{X}_{i}, \ddot{X}_{i}\right)$ is also nonlinear function of the responses of TLP. Hence the wave loads on TLP are coupled with the dynamic responses. This nonlinear situation differs from that of the linear one, in which the acceleration and velocity of fluid particle, the unit vector $\boldsymbol{e}_{3}$ are all evaluated at the original still position of TLP and have nothing to do with the displacements of structure.

Integrating along the column, we can obtain the hydrodynamic force vector acting on the whole column (column 1):

$$
\boldsymbol{F}_{\mathrm{w} 1}=\int_{-h_{G}}^{-h_{G}+h_{1}} \boldsymbol{f}_{\mathrm{n} 1} d \zeta=\boldsymbol{F}_{\mathrm{w} 1}\left(X_{i}, \dot{X}_{i}, \ddot{X}_{i}\right),
$$

where $h_{G}$ is the distance between C.G. and the bottom of cylinder, and $h_{1}$ is the distance along centerline of column 1 from the bottom of column to the instantaneous wetted surface at any time. $\boldsymbol{f}_{\mathrm{n} 1}$ is $\boldsymbol{f}_{\mathrm{n}}$ on column 1 . The moment vector $\boldsymbol{M}_{\mathrm{Gw} 1}$ with reference to the principal axes of TLP generated by hydrodynamic force is given as follows:

$$
\boldsymbol{M}_{\mathrm{Gw} 1}=\int_{-h_{G}}^{-h_{G}+h_{1}}\left(\boldsymbol{r}_{G} \times \boldsymbol{f}_{\mathrm{n} 1} d \zeta\right)=\boldsymbol{M}_{G \mathrm{w} 1}\left(X_{i}, \dot{X}_{i}, \ddot{X}_{i}\right) .
$$




\subsection{Hydrostatic force vector}

As the buoyancy $\boldsymbol{F}_{B}$ is always perpendicular to still water surface, the hydrostatic force on TLP is only in the heave direction. The magnitude of buoyancy is

$$
\left|\boldsymbol{F}_{B}\right|=\rho g \pi r^{2} \cdot\left(h_{1}+h_{2}+h_{3}+h_{4}\right)+\rho g D_{a y} D_{a z} \cdot 4 a+\rho g D_{b x} D_{b z} \cdot 4 b,
$$

where $D_{a y}, D_{a z}, D_{b x}, D_{b z}, a, b$ are shown in Fig.1. In this paper, $h_{i}(i=1,2,3,4)$ are always altering and evaluated at the instantaneous position of TLP. Therefore $h_{i}$ are nonlinear functions of displacements and wave elevation, i.e., $h_{i}=h_{i}\left(X_{i}\right)$. The buoyancy can be expressed as

$$
\left|\boldsymbol{F}_{B}\right|=\left|\boldsymbol{F}_{B}\left(X_{i}\right)\right| \text {. }
$$

While for the situation of small displacement, $h_{i}$ is evaluated at the still position and independent of the wave elevation.

When TLP rotates, the center of buoyancy will depart from the symmetry axis. Consequently the buoyancy will generate moment with reference to the C.G. The buoyancy and buoyancy generating moment vectors on column 1 are given below, and those on the other columns and pontoons can be obtained similarly.

The magnitude of buoyancy on column 1 is $\boldsymbol{F}_{B 1}\left(X_{i}\right)=\rho g \pi r^{2} h_{1}$, pointing upward perpendicular to the still water surface. $r$ is the radius of column. The coordinates of shifted center of buoyancy in $G \xi \eta \zeta$ are $\left(B_{\xi}, B_{\eta}, B_{\zeta}\right)$,

$$
B_{\xi}=-\frac{r^{2} t_{31}}{4 h_{1} t_{33}}+a, \quad B_{\eta}=-\frac{r^{2} t_{32}}{4 h_{1} t_{33}}-b, \quad B_{\zeta}=\frac{h_{1}}{2}+\frac{r^{2}\left(t_{31}^{2}+t_{32}^{2}\right)}{8 h_{1} t_{33}^{2}}-h_{G} .
$$

The moment vector induced by buoyancy on column 1 is

$$
\boldsymbol{M}_{G B 1}=\boldsymbol{r}_{G B} \times \boldsymbol{F}_{B 1}=\boldsymbol{M}_{G B 1}\left(X_{i}\right),
$$

where $\boldsymbol{r}_{G B}$ is the position vector of center of buoyancy with respect to C.G. It is obvious that $\boldsymbol{F}_{B 1}\left(X_{i}\right)$ and $\boldsymbol{M}_{G B 1}\left(X_{i}\right)$ are both nonlinear functions of displacements.

\subsection{Tension vector in tendon}

The existing method for deriving the tension of tendon is only suitable for linear case because the stiffness matrix is derived with respect to the initial still equilibrium position. Hence the stiffness matrix is constant matrix and the tension is linear function of the displacements of TLP. Whereas the stiffness matrix should be derived with reference to the instantaneous position as the finite displacements are taken into account, i.e., the stiffness matrix is function of the displacements and all of the six degrees of freedom should be simultaneously considered. In this paper, we express the tension in terms of the displacements of six degrees of freedom instead of by stiffness matrix. It will induce the coupling among the six degrees of freedom, which is apparently another type of nonlinearity.

We also illustrate the deriving of related formulas by tendon 1. Point $A$ is at the bottom of column 1, point $B$ is fixed at the seabed. When TLP moves to arbitrary position, the coordinates of points $A$ and $B$ are $\left(A_{X}, A_{Y}, A_{Z}\right)$ and $\left(B_{X}, B_{Y}, B_{Z}\right)$, respectively, where

$$
\left(A_{X}, A_{Y}, A_{Z}\right)^{\mathrm{T}}=\left(\begin{array}{l}
X_{1}+t_{11} a-t_{12} b-t_{13} h_{G} \\
X_{2}+t_{21} a-t_{22} b-t_{23} h_{G} \\
X_{3}+t_{31} a-t_{32} b-t_{33} h_{G}
\end{array}\right), \quad\left(B_{X}, B_{Y}, B_{Z}\right)^{\mathrm{T}}=\left(\begin{array}{c}
a \\
-b \\
-\left(h_{G}+L\right)
\end{array}\right),
$$

$L$ is the initial length of the tendon. Then the tension vector of tendon 1 can be given as

$$
\boldsymbol{F}_{t 1}=\left(T_{0}+\frac{E S}{L}\left(L_{1}-L\right)\right) \cdot \frac{\boldsymbol{A} \boldsymbol{B}}{|\boldsymbol{A B}|}=\boldsymbol{F}_{t 1}\left(X_{i}\right),
$$


in which $T_{0}$ is the initial pretension in the tendon, $E$ is Young's Modulus, $S$ is the crosssectional area of the tendon, $L_{1}(=|\boldsymbol{A} \boldsymbol{B}|)$ is the instantaneous length of the tendon. Moreover, the moment vector $\boldsymbol{M}_{G t 1}$ with reference to the C.G. induced by tension is given as follows:

$$
\boldsymbol{M}_{G t 1}=\boldsymbol{r}_{G A} \times \boldsymbol{F}_{t 1}=\boldsymbol{M}_{G t 1}\left(X_{i}\right),
$$

where $\boldsymbol{r}_{G A}$ is the position vector of point $A$ with respect to C.G. $\boldsymbol{F}_{t 1}\left(X_{i}\right), \boldsymbol{M}_{G t 1}\left(X_{i}\right)$ are both nonlinear functions of the displacements. Similarly, the tension and moment vectors of the other three tendons can also be obtained.

In the above several sections, we have given formulas for calculating the forces and moments acting on column 1. The formulas for other columns and pontoons are similar and easy to acquire so long as the respective position vectors and direction vectors of the referred structures are substituted. Summing the forces and moments vectors on all columns and pontoons, we can obtain the overall external forces and moments. Moreover, we can begin to solve Eq.(2).

\section{Numerical solution of motion equation}

Now the external forces and moments are all nonlinear functions of the responses of TLP on condition that the displacements are finite, the motion equation (2) of TLP is the coupled nonlinear differential equations, which can hardly be solved analytically. In this paper, we adopt numerical method. Substituting Eqs.(3)-(5) and Eqs.(3a)-(5a) into Eq.(2), we can see that Eq.(2) are 6 unknowns second-order ordinary differential equations with respect to 6 degrees of freedom $X_{i}$. After performing order reduction of the equations, we can transform Eq.(2) into first-order differential equations in 12 unknowns. Then the standard format of RungeKutta numerical time integration procedure can be followed to program the computer to solve the equations. In this paper, a fourth-order Runge-Kutta method with constant time step is adopted. The computer program for numerical solution is written with FORTRAN language.

To verify the proposed method in this paper and the computer program, we carry out the dynamic responses calculation for a typical TLP (ISSC TLP) by our program. The primary properties of ISSC TLP are shown in Table 1.

Table 1 Primary properties of ISSC TLP ${ }^{[11]}$

\begin{tabular}{cccc}
\hline $\begin{array}{c}\text { Spacing between column } \\
86.25 \mathrm{~m}\end{array}$ & Column radius & Pontoon width & Pontoon height \\
& $8.44 \mathrm{~m}$ & $7.5 \mathrm{~m}$ & $10.5 \mathrm{~m}$ \\
\hline Draft & Displacement & Mass & Length of tendons \\
$35.0 \mathrm{~m}$ & $54.5 \times 10^{6} \mathrm{~kg}$ & $40.5 \times 10^{6} \mathrm{~kg}$ & $415.0 \mathrm{~m}$ \\
\hline Roll moment of inertia & Pitch moment of inertia & Yaw moment of inertia & Height of C.G. above keel \\
$82.37 \times 10^{9} \mathrm{~kg} \cdot \mathrm{m}^{2}$ & $82.37 \times 10^{9} \mathrm{~kg} \cdot \mathrm{m}^{2}$ & $98.07 \times 10^{9} \mathrm{~kg} \cdot \mathrm{m}^{2}$ & $38.0 \mathrm{~m}$ \\
\hline
\end{tabular}

The RAOs of ISSC TLP under the circumstances of first order small displacements are computed, which is the degenerative case of the finite displacements. The displacements RAOs of the TLP in directions of surge, sway and yaw in regular waves with heading angle of $22.5^{\circ}$ are compared with the existing solution and shown in Figs.2-4. The hollow points represent the first order small displacements linear solution of this paper, while the solid points represent the counterparts by Taylor and Jefferys ${ }^{[11]} . X_{1 a}, X_{2 a}, X_{6 a}$ are amplitudes of surge, sway and yaw, respectively. $H$ and $T_{\mathrm{w}}$ are the wave height and wave period, respectively. It can be easily found that our degenerative linear solutions coincide with that of existing solutions.

Then the dynamic responses of ISSC TLP taking account of multifold nonlinearities induced by finite displacements are computed, and compared with that of linear case. The linear and nonlinear solutions of the steady state responses of ISSC TLP in regular waves are shown in 


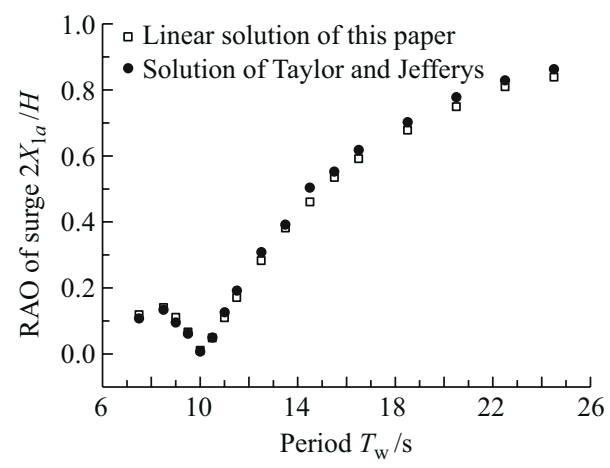

Fig.2 RAO of surge

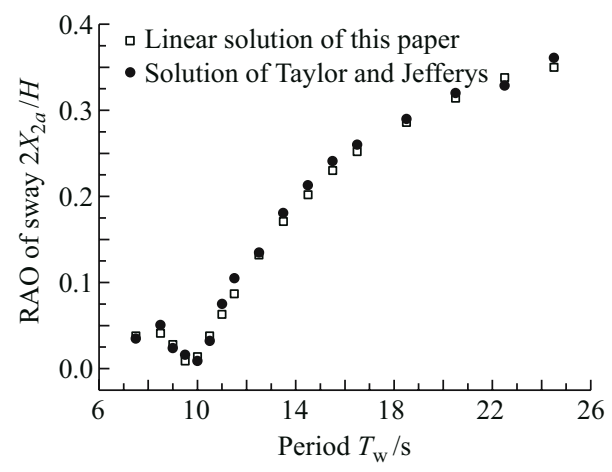

Fig.3 RAO of sway

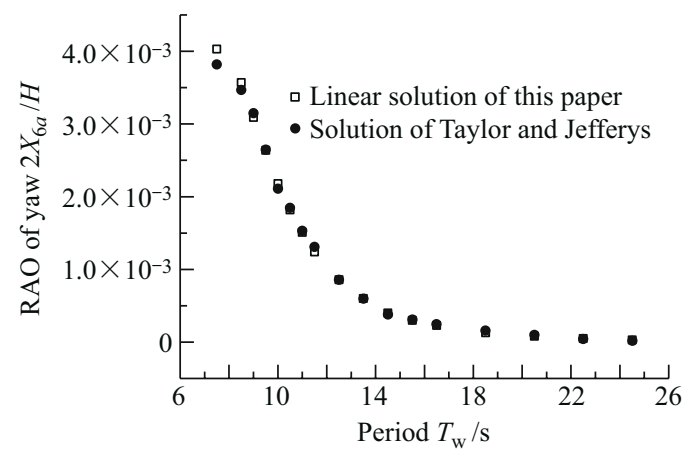

Fig.4 RAO of yaw

Figs.5-10. The wave heading angle is $22.5^{\circ}$, and the wave heights are $8 \mathrm{~m}$ and $11 \mathrm{~m}$ respectively. It can be found that nonlinearities exert a distinct influence on the dynamic responses of TLP, and the differences between linear and nonlinear solutions become more distinct as the wave height increases. The linear solutions of surge, sway and yaw have nearly zero net excursions, whereas the nonlinear solutions reveal obvious net excursions. For the waves considered in the paper, the amplitude differences between those two type solutions of surge and sway are about $20 \% \sim 67 \%$, and the differences of yaw are about $4 \% \sim 7 \%$. The phase of nonlinear solutions of heave is shifted from that of linear one by about $180^{\circ}$, and the amplitude differences are about $70 \%$. The differences between the linear and nonlinear solutions of roll and pitch are very large, which can be multiple times. In addition, the presence of high-frequency components in the nonlinear solutions of roll and pitch is apparent. The foregoing differences between linear and nonlinear solutions may be attributed to both the complicated nonlinear coupling among six degrees of freedom (e.g., the change of surge can induce the evident variety of heave and rotations, while in small displacements case it will not.) and the loads-responses interactions induced by finite displacements (i.e., loads are nonlinear functions of responses). The net excursion (drift), high-frequency components, amplitude differences and phase shifts therein mentioned are typical consequences induced by nonlinear terms (e.g., the quadratic, cubic and high order terms introduced by finite displacements) in the governing equations.

In order to investigate the variance of the difference between the linear and nonlinear solutions as the wave height decreases, we calculate the dynamic responses of ISSC TLP in regular waves with wave height being $5 \mathrm{~m}, 4 \mathrm{~m}, 3 \mathrm{~m}, 2 \mathrm{~m}$, respectively. It is shown that, as the wave 
height
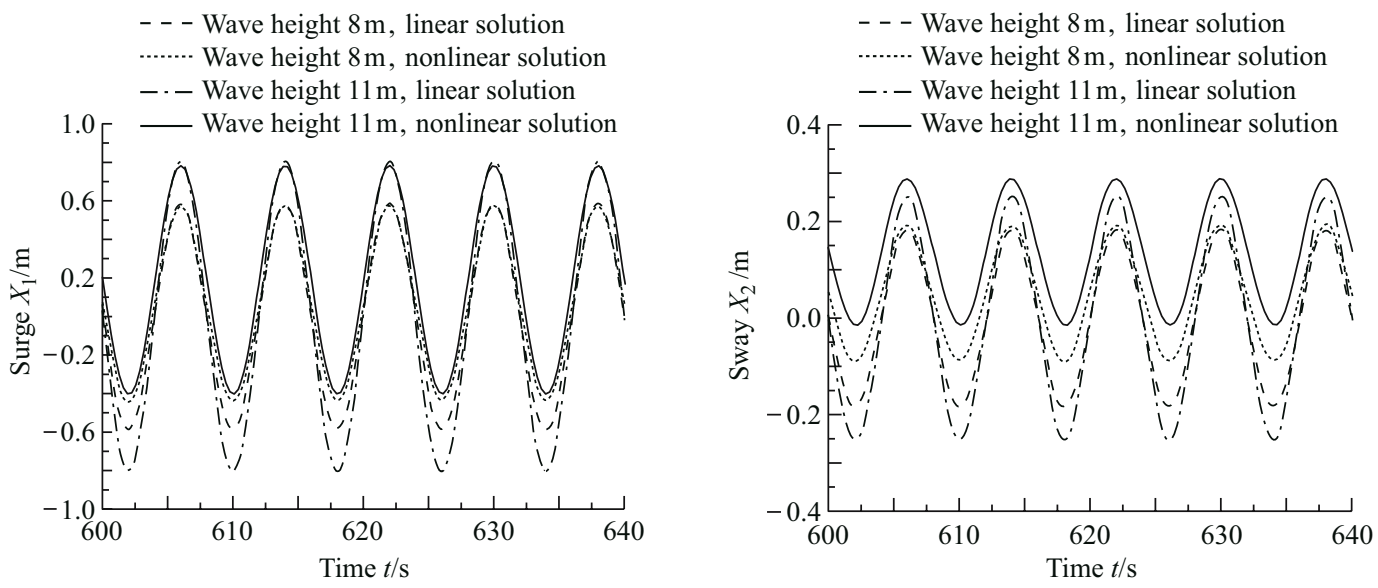

Fig.5 Steady-state response of surge $(8 \mathrm{~m}, 11 \mathrm{~m})$ Fig.6 Steady-state response of sway $(8 \mathrm{~m}, 11 \mathrm{~m})$

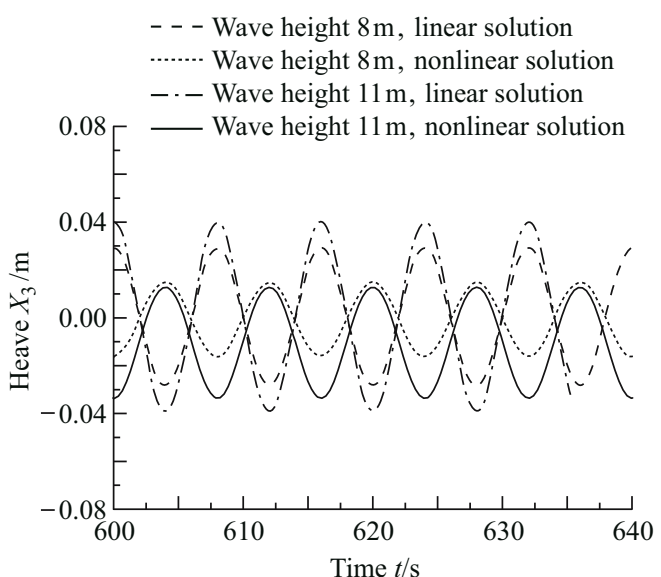

Fig.7 Steady-state response of heave $(8 \mathrm{~m}, 11 \mathrm{~m})$



Fig.8 Steady-state response of $\operatorname{roll}(8 \mathrm{~m}, 11 \mathrm{~m})$

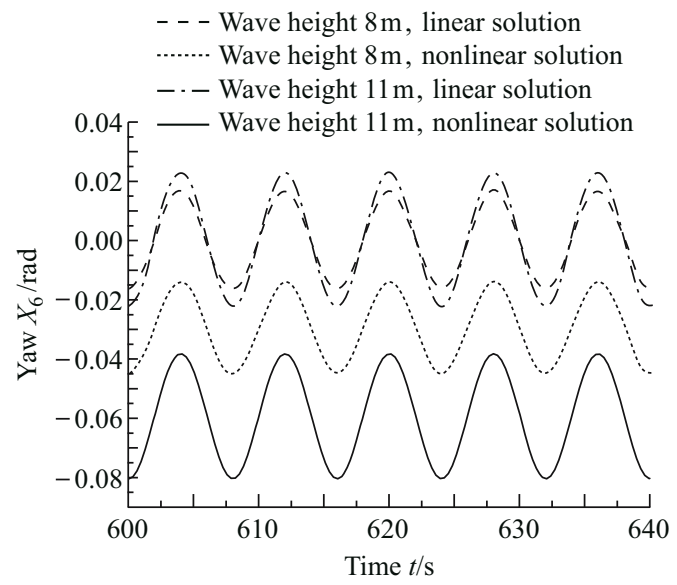

Fig.9 Steady-state response of pitch $(8 \mathrm{~m}, 11 \mathrm{~m})$ Fig.10 Steady-state response of yaw $(8 \mathrm{~m}, 11 \mathrm{~m})$ 
decreases, the linear and nonlinear solutions tend to coincide with each other. The differences of those two type solutions of surge are lesser than that of sway, because the surge direction and the wave propagation direction form an angle different from that of the sway direction. Although the differences of the linear and nonlinear solutions of heave, roll, pitch and yaw also diminish as the wave height decreases, they do not diminish distinctly as that of surge and sway. The intrinsic characteristics such as phase difference, high-frequency components and net excursion of heave, roll, pitch and yaw, which nonlinear solutions differ from linear solutions as mentioned above, do not disappear as wave height decreases. The amplitude difference of linear and nonlinear solutions of surge and sway are listed in Table 2, where wave heights are $5 \mathrm{~m}$, $4 \mathrm{~m}, 3 \mathrm{~m}, 2 \mathrm{~m}$, respectively. The linear and nonlinear solutions of the steady state responses of ISSC TLP in regular waves with wave height being $5 \mathrm{~m}, 2 \mathrm{~m}$, respectively, are shown in Figs.11-16.

If only the two relatively large displacements (surge and sway) are concerned, the linear solutions can be adopted in mild sea states. However, if the other four relatively small displacements are also concerned, though the sea state is mild, nevertheless the nonlinear solutions should be adopted. The reason is that though the wave heights are small, the nonlinear solutions of heave, roll, pitch and yaw still differ from linear solutions distinctly.
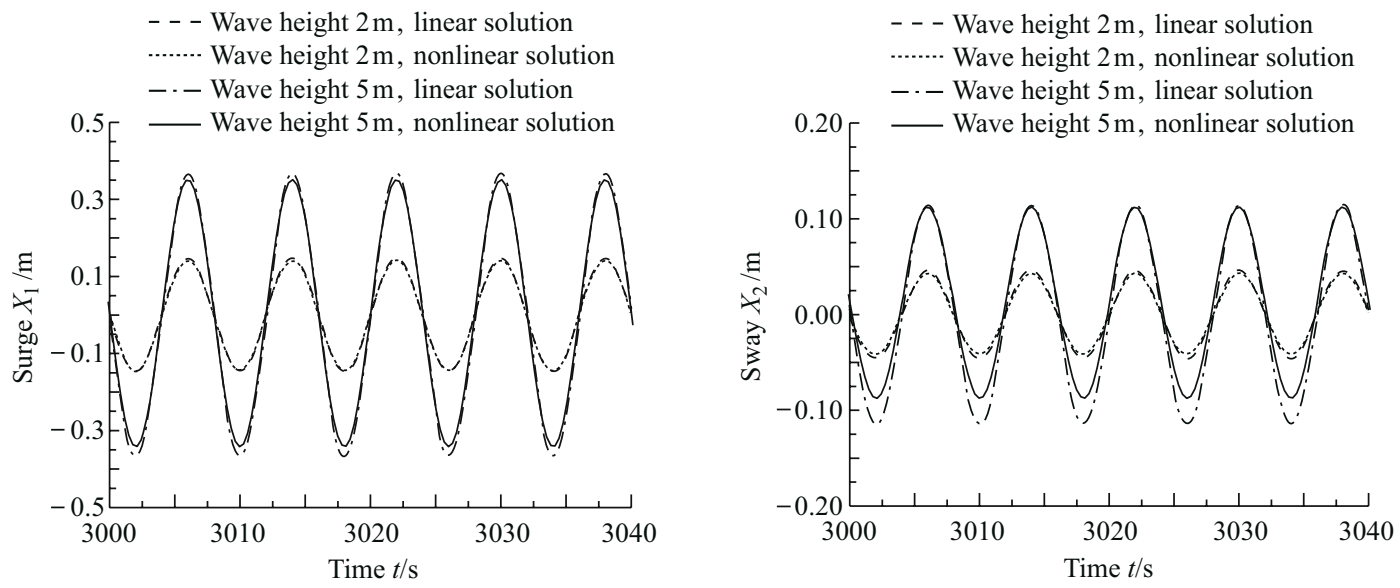

Fig.11 Steady-state response of surge $(2 \mathrm{~m}, 5 \mathrm{~m})$ Fig.12 Steady-state response of sway (2 m, $5 \mathrm{~m})$
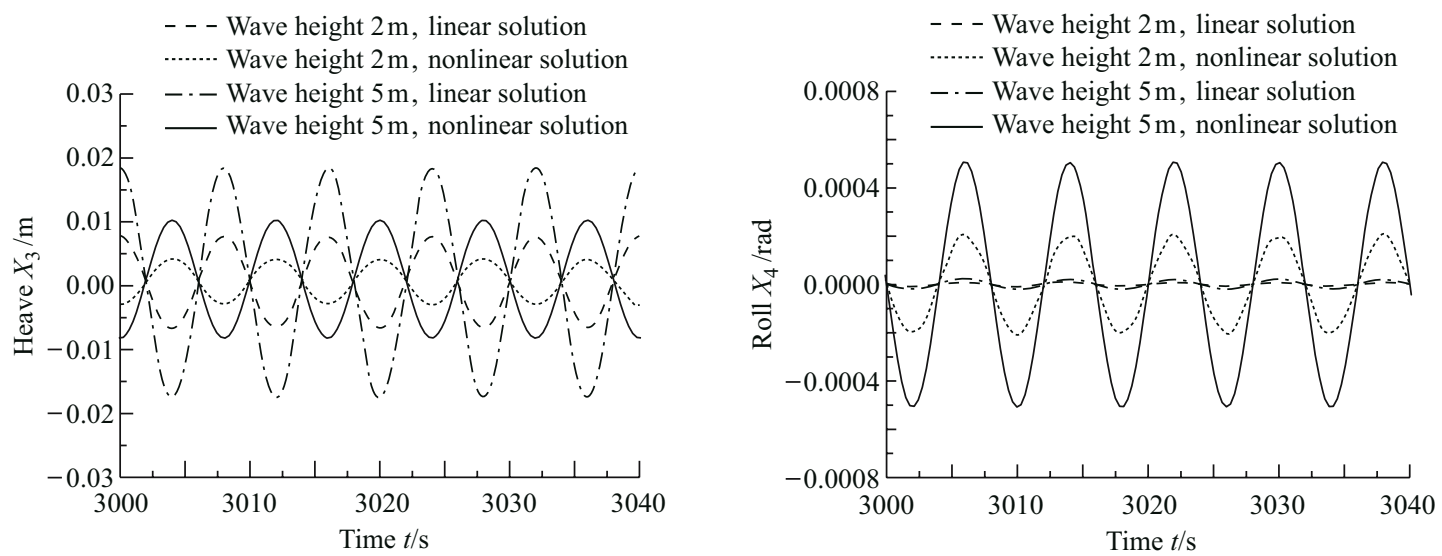

Fig.13 Steady-state response of heave $(2 \mathrm{~m}, 5 \mathrm{~m})$ Fig.14 Steady-state response of roll $(2 \mathrm{~m}, 5 \mathrm{~m})$ 

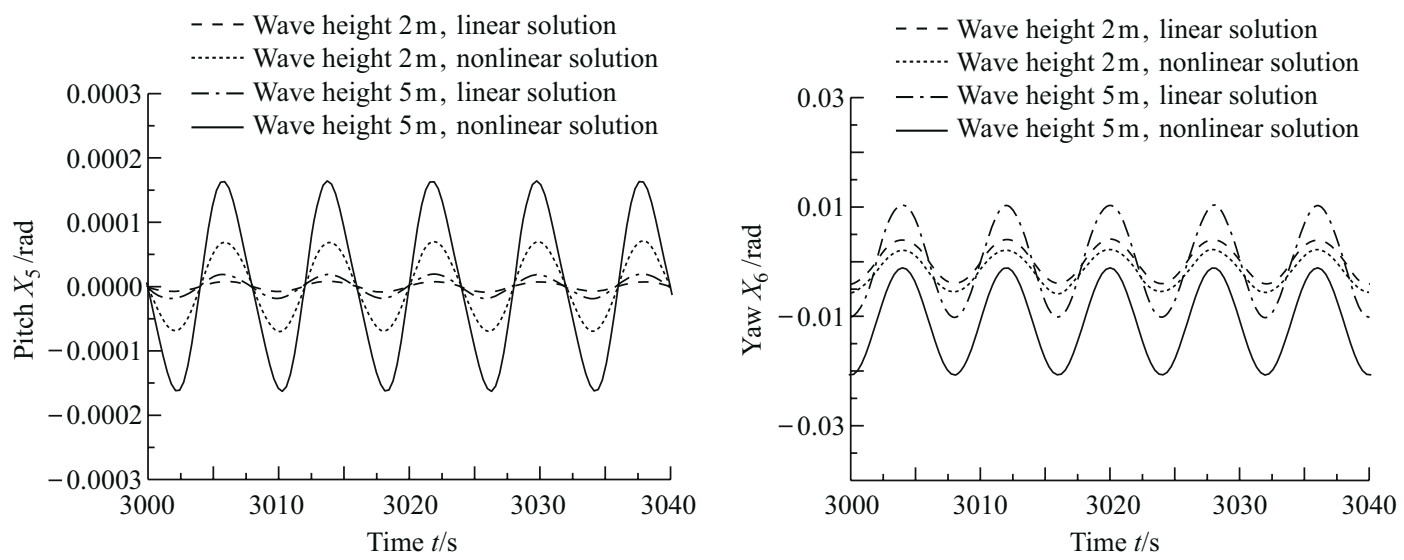

Fig.15 Steady-state response of pitch $(2 \mathrm{~m}, 5 \mathrm{~m})$ Fig.16 Steady-state response of yaw $(2 \mathrm{~m}, 5 \mathrm{~m})$

Table 2 Differences of linear and nonlinear solutions as wave height decreases (wave period $8 \mathrm{~s}$, wave heading angle $22.5^{\circ}$ )

\begin{tabular}{ccccc}
\hline Wave height $/ \mathrm{m}$ & 5 & 4 & 3 & 2 \\
\hline Differences of surge/\% & 5.7 & 3.9 & 1.9 & 1.3 \\
Differences of sway $/ \%$ & 13.0 & 10.4 & 8.7 & 5.9 \\
\hline
\end{tabular}

\section{Conclusions}

The dynamic responses of TLP with finite displacements are investigated in this paper. Several nonlinearities induced by finite displacements are taken into account. The differences between nonlinear and linear cases are clarified. Then a method for computing dynamic responses of TLP considering multifold nonlinearities is proposed. The nonlinear factors include finite displacement, coupling of the six degrees of freedom, instantaneous position, instantaneous wet surface, free surface effects and viscous drag force. The formulas are given, and a computer program for numerical analysis is developed by using FORTRAN language.

To verify the proposed method, a degenerative linear case for ISSC TLP is computed and compared with the published one, which shows good agreements. Moreover, the comparison between the nonlinear and linear cases shows significant differences. Based on the numerical case study in this paper, it reveals that nonlinear factors play an important role in the dynamic response of all the 6 degrees of freedom of TLP when the wave height is large. When the wave height is small, the differences of linear and nonlinear solutions of surge and sway are comparatively unapparent; while the differences of linear and nonlinear solutions of heave, roll, pitch and yaw are still distinct. Therefore, it is suggested that the nonlinearities deserve a serious thought, especially for severe sea state. Some validations may be needed to perform before any approximation related to nonlinear factors referred in this paper is made.

Acknowledgements We gratefully acknowledge the beneficial advice by Professor LI Jia-chun.

\section{References}

[1] Williams A N, Rangappa T. Approximate hydrodynamic analysis of multicolumn ocean structures[J]. Ocean Engineering, 1994, 21(6):519-573. 
[2] Yilmaz O. Hydrodynamic interactions of waves with group of truncated vertical cylinders[J]. Journal of Waterway, Port, Coastal, and Ocean Engineering, 1998, 124(5):272-279.

[3] Yilmaz O, Incecik A, Barltrop N. Wave enhancement due to blockage in semi-submersible and TLP structures[J]. Ocean Engineering, 2001, 28(5):471-490.

[4] Ahmad S. Stochastic TLP response under long crested random sea[J]. Computers and Structures, 1996, 61(6):975-993.

[5] Ahmad S, Islam N, Ali A. Wind-induced response of tension leg platform[J]. Journal of Wind Engineering and Industrial Aerodynamics, 1997, 72(1-3):225-240.

[6] Chandrasekaran S, Jain A K. Dynamic behaviour of square and triangular offshore tension leg platforms under regular wave loads[J]. Ocean Engineering, 2002, 29(3):279-313.

[7] Chandrasekaran S, Jain A K. Triangular configuration tension leg platform behaviour under random sea wave loads[J]. Ocean Engineering, 2002, 29(15):1895-1928.

[8] Zeng Xiaohui, Shen Xiaopeng, Wu Yingxiang. Nonlinear dynamic response of floating circular cylinder with taut tether[C]. In: Jin S, Chun G (eds). Proceedings of the 15th International Offshore and Polar Engineering Conference, California: ISOPE, 2005, 1:218-224.

[9] Clauss G, Lehmann E, Ostergaard C. Offshore structures[M]. London: Springer-Verlag, 1992.

[10] Chakrabarti S K. Hydrodynamics of offshore structures[M]. Southampton: Computational Mechanics Publications, 1987.

[11] Taylor R E, Jefferys E R. Variability of hydrodynamic load predictions for a tension leg platform[J]. Ocean Engineering, 1986, 13(5):449-490. 